\title{
Conditional Lie-Bäcklund Symmetries and Reductions of the Nonlinear Diffusion Equations with Source
}

\author{
Junquan Song, ${ }^{1}$ Yujian Ye, ${ }^{2}$ Danda Zhang, ${ }^{1}$ and Jun Zhang ${ }^{1}$ \\ ${ }^{1}$ Department of Applied Mathematics, Zhejiang University of Technology, Hangzhou 310023, China \\ ${ }^{2}$ School of Management, Hangzhou Dianzi University, Hangzhou 310018, China \\ Correspondence should be addressed to Junquan Song; mathjqsong@163.com
}

Received 10 October 2013; Revised 16 December 2013; Accepted 25 December 2013; Published 25 February 2014

Academic Editor: Weiguo Rui

Copyright (c) 2014 Junquan Song et al. This is an open access article distributed under the Creative Commons Attribution License, which permits unrestricted use, distribution, and reproduction in any medium, provided the original work is properly cited.

Conditional Lie-Bäcklund symmetry approach is used to study the invariant subspace of the nonlinear diffusion equations with source $u_{t}=e^{-q x}\left(e^{p x} P(u) u_{x}^{m}\right)_{x}+Q(x, u), m \neq 1$. We obtain a complete list of canonical forms for such equations admit multidimensional invariant subspaces determined by higher order conditional Lie-Bäcklund symmetries. The resulting equations are either solved exactly or reduced to some finite-dimensional dynamic systems.

\section{Introduction}

The classical symmetry theory for studying differential equations is presented firstly by Lie, which has been universally used and proved to be very effective in similarity reductions and group classifications [1-7]. However, there exist some important equations with very small Lie point symmetry groups. For example, the Fisher equation and FitzhughNagumo equation, which are widely used in mathematical biology, are invariant only under the time and space translations. This means that the classical symmetry reduction method is not a proper tool for dealing with these equations. To overcome this difficulty, several generalized methods have been developed and established, including the nonclassical symmetry method (or referred to as the conditional symmetry method) [8], the weak symmetry method $[9,10]$, iteration of the nonclassical method [11], the Clarkson-Kruskal direct method $[12,13]$, and the conditional Lie-Bäklund symmetry (CLBS) method (or referred to as the generalized conditional symmetry method) [14-16].

CLBS can be regarded as a natural generalization of the nonclassical symmetry. Therefore, the procedure for computing the CLBSs is about the same as for the nonclassical method. Furthermore, Galaktionov and Svirshchevski have shown that the CLBS method is closely related to the invariant subspace (IS) method; namely, exact solutions defined on ISs for differential equations or their variant forms can be obtained by using the CLBS method [17-19]. For nonlinear diffusion equations (NLDEs), symmetry-related methods, especially the CLBS method, have been proved to be very powerful to classify and reduce the considered equations [2034]. For example, NLDEs

$$
f(x) u_{t}=\left(g(x) w(u) u_{x}\right)_{x}
$$

can be used to describe not only the process by which matter is transported from one part of a system to another, as a result of random molecular motion, but they can also represent many other apparently unrelated phenomena such as heat conduction in solids or even the stationary notion of a boundary layer of fluid over a flat plate [35]. In [36], the Lie point symmetry method has been used to obtain the similarity solutions of the inhomogeneous NLDEs

$$
u_{t}=x^{-q}\left(x^{p} u^{n} u_{x}\right)_{x}
$$

belonging to the above equations, where nonzero constants $p$ and $q$ have several applications such as propagation of a thermal wave in an exponential atmosphere. A complete classification of the symmetry reductions of these equations using the nonclassical method is given by Saied in [37]. The secondorder CLBSs of these equations have also been studied in [33]. Furthermore, the generalized porous medium equations

$$
u_{t}=\left(P(u) u_{x}^{m}\right)_{x}+Q(u), \quad m \neq 1,
$$


are considered by using the CLBS method in [34]. Some exact solutions, defined on the polynomial, trigonometric, and exponential ISs determined by the CLBSs, are constructed.

In this paper, we mainly discuss the following NLDEs:

$$
u_{t}=e^{-q x}\left(e^{p x} P(u) u_{x}^{m}\right)_{x}+Q(x, u), \quad m \neq 1,
$$

by means of the CLBS method. Here, $P(u)$ and $Q(x, u)$ are, respectively, referred to as the diffusion and source terms. Equation (4) has a wide range of applications in physics, diffusion process, and engineering sciences and has been applied to describe several situations such as heat conduction by electrons in a plasma, heat conduction by radiation in a fully ionized gas, axisymmetric flow of a very viscous fluid, and turbulent diffusion [38, 39].

The remainder of this paper is organized as follows. In the following section, we review some necessary notations, definitions, and fundamental theorems on the CLBS method. Equations of the form (4) admitting CLBSs and the corresponding ISs are classified in Section 3. Exact solutions and reductions of some examples in the resulting equations are obtained in Section 4. The last section is devoted to conclusions and discussions.

\section{Preliminaries}

Let us give a brief discussion on the CLBS method. For the $m$ th-order equation

$$
u_{t}=E\left(t, x, u, u_{1}, \ldots, u_{m}\right),
$$

we set

$$
V=\sum_{k=0}^{\infty} D_{x}^{k} \eta \frac{\partial}{\partial u_{k}}
$$

as an evolutionary vector field with characteristic $\eta$. Here, we use the following notations:

$$
\begin{gathered}
D_{x}=\frac{\partial}{\partial x}+\sum_{k=0}^{\infty} u_{k+1} \frac{\partial}{\partial u_{k}}, \quad D_{x}^{j+1}=D_{x}\left(D_{x}^{j}\right), \\
D_{x}^{0}=1, \quad u_{k}=\frac{\partial^{k} u}{\partial x^{k}} .
\end{gathered}
$$

Definition 1. The evolutionary vector field (6) is said to be a Lie-Bäcklund symmetry of (5) if and only if

$$
\left.V\left(u_{t}-E\right)\right|_{L}=0,
$$

where $L$ is the set of all differential consequences of the equation; that is,

$$
u_{t}-E=0, \quad D_{x}^{j} D_{t}^{k}\left(u_{t}-E\right)=0, \quad j, k=0,1,2, \ldots
$$

Definition 2. The evolutionary vector field (6) is said to be a CLBS of (5) if and only if

$$
\left.V\left(u_{t}-E\right)\right|_{L \cap M}=0,
$$

where $M$ denotes the set of all differential consequences of equation $\eta=0$ with respect to $x$; that is, $D_{x}^{j} \eta=0$, $j=0,1,2, \ldots$.
Proposition 3 (Zhdanov [14] and Fokas and Liu [15, 16]). Equation (5) admits the CLBS (6) if there exists a function $W(t, x, u, \eta)$ such that

$$
\frac{\partial \eta}{\partial t}=[E, \eta]+W(t, x, u, \eta), \quad W(t, x, u, 0)=0,
$$

where $[E, \eta]=E^{\prime} \eta-\eta^{\prime} E$, the prime denotes the Fréchet derivative, and $W$ is an analytic function of $t, x, u, u_{1}$, and $\eta, D_{x} \eta$, $D_{x}^{2} \eta, \ldots$.

An obvious conclusion of this proposition is that (5) admits the CLBS with the characteristic $\eta$ if

$$
\left.D_{t} \eta\right|_{L \cap M}=0
$$

Here, $L$ and $M$ are given as in Definitions 1 and 2 .

For (4), we set the characteristic

$$
\sigma=[g(u)]_{l x}+a_{1}(x)[g(u)]_{(l-1) x}+\cdots+a_{l}(x) g(u),
$$

where $[g(u)]_{i x}=\partial^{i}[g(u)] / \partial x^{i}, i=1,2, \ldots, l$, and $l \in N$. It is important to note that if (4) admits CLBS (13), then equation

$$
\begin{aligned}
v_{t}= & e^{(p-q) x}\left[A(v) v_{x}^{m-1}\left(v_{x x}+\frac{p}{m} v_{x}\right)+B(v) v_{x}^{m+1}\right] \\
& +C(x, v)
\end{aligned}
$$

admits CLBS

$$
\eta=v_{l x}+a_{1}(x) v_{(l-1) x}+\cdots+a_{l}(x) v .
$$

In fact, (4) and (14) are related as

$$
\begin{gathered}
A(v)=m P\left[f(v), f^{\prime}(v)\right]^{m-1}, \\
B(v)=\frac{1}{m}\left[\frac{f^{\prime \prime}(v)}{f^{\prime}(v)} A(v)+A^{\prime}(v)\right], \\
C(x, v)=\frac{Q(x, f(v))}{f^{\prime}(v)},
\end{gathered}
$$

where $u=f(v)$ denotes the inverse function of $v=g(u)$.

From (12), we can see that (14) admitting of the CLBS with the characteristic (15) is equivalent to $\left.\eta^{\prime} E[v]\right|_{M}=0$; namely,

$$
(E[v])_{l x}+a_{1}(x)(E[v])_{(l-1) x}+\cdots+\left.a_{l}(x)(E[v])\right|_{M}=0,
$$

where

$$
\begin{aligned}
E[v] \equiv & e^{(p-q) x}\left[A(v) v_{x}^{m-1}\left(v_{x x}+\frac{p}{m} v_{x}\right)+B(v) v_{x}^{m+1}\right] \\
& +C(x, v)
\end{aligned}
$$

and $M$ is given as in Definition 2. Thus, the linear solution space

$$
W_{l}=W\left\{f_{1}(x), f_{2}(x), \ldots, f_{l}(x)\right\}
$$


of linear ordinary differential equation (ODE) $\eta=0$ is invariant with respect to the above operator $E[v]$; that is,

$$
E\left[\sum_{i=1}^{l} C_{i} f_{i}(x)\right]=\sum_{i=1}^{l} \Psi_{i}\left(C_{1}, C_{2}, \ldots, C_{l}\right) f_{i}(x) .
$$

It follows that if (14) admits CLBS (15), then (14) has an exact solution of the generalized separation of variables form

$$
v(x, t)=\sum_{i=1}^{l} C_{i}(t) f_{i}(x),
$$

where the coefficients $C_{i}(t)$ satisfy the $l$-dimensional dynamic system

$$
C_{i}^{\prime}(t)=\Psi_{i}\left(C_{1}(t), C_{2}(t), \ldots, C_{l}(t)\right), \quad i=1,2, \ldots, l .
$$

Thus, we will determine the forms of (14) so that (14) admits the CLBS (15), which is equivalent to classify (14) in terms of the IS (19), or it is equivalent to study the CLBS (13) of (4).

The following theorem provides us with the estimate to the maximal dimension of the IS admitted by an $m$ th-order nonlinear differential operator.

Theorem 4 (Galaktionov and Svirshchevski [19]). If a linear space $W_{l}$ is invariant with respect to the nonlinear differential operator $E$ of order $m$, then there exists an inequality

$$
l \leq 2 m+1 .
$$

It follows that the order of linear ODE $\eta=0$ is not greater than five if (14) admits the CLBS (15). This allows us to classify (14) based on the existence of the generalized variable separation solutions (GVSSs) (21), which are generated by the solution space (19) determined by the linear ODE $\eta=0$, or it is equivalent to study the GVSSs $u=f(v)$ of (4) generated by $\sigma=0$.

\section{CLBSs and Corresponding ISs of (14)}

In view of Theorem 4 , it suffices to consider CLBSs (15) of (14) with $2 \leq l \leq 5$. We first consider the case $l=2$. It implies from (17) that (14) admits the CLBS (15) if there holds

$$
\begin{aligned}
& \left.\eta^{\prime} E[v]\right|_{M} \\
& =e^{(p-q) x} B^{\prime \prime} v_{x}^{m+3}+e^{(p-q) x} \\
& \times\left\{\left[\frac{p}{m}-a_{1}\right] A^{\prime \prime}+2\left[p-q-(m+1) a_{1}\right] B^{\prime}\right\} v_{x}^{m+2} \\
& +e^{(p-q) x}\left\{m\left(2 A^{\prime}+(m+1) B\right) a_{1}^{2}-\left[(m+1) B+2 A^{\prime}\right] a_{1}^{\prime}\right. \\
& -\left[A^{\prime \prime} v+2 A^{\prime}+(2 m+3) B^{\prime} v+m B\right] a_{2}^{\prime} \\
& +p\left[\frac{2}{m}(p-q)-2 a_{1}\right] A^{\prime}+(p-q) \\
& \left.\times\left[B\left(p-(2 m+1) a_{1}\right)-2 a_{1} A^{\prime}\right]+B q^{2}\right\} v_{x}^{m+1}
\end{aligned}
$$

$$
\begin{aligned}
& +e^{(p-q) x}\left\{-\left(m^{2}-m\right) A a_{1}^{3}+[(3 m-2) p-(2 m-1) q]\right. \\
& \times A a_{1}^{2}+\left[(4 m-1) A^{\prime} v+2 m(m+1) B v\right. \\
& +3(m-1) A] a_{1} a_{2} \\
& +\left[-(p-q)\left(\frac{3 m-1}{m} p-q\right)+(3 m-1) a_{1}^{\prime}\right] A a_{1} \\
& +\left[-\frac{(2 m+1) A^{\prime} v+(m-1) A}{m} p\right. \\
& \left.-2(p-q)\left(A+A^{\prime} v+(2 m+1) B v\right)\right] a_{2} \\
& -\left(2 A^{\prime} v+2 A+(m+1) B v\right) a_{2}^{\prime} \\
& \left.+\left[\frac{p(p-q)^{2}}{m}-a_{1}^{\prime \prime}+(2 q-3 p) a_{1}^{\prime}\right] A\right\} v_{x}^{m} \\
& +e^{(p-q) x}\left\{-(3 m-2)(m-1) A a_{1}^{2} a_{2} v\right. \\
& +[(6 m-5) p-(4 m-3) q] A v a_{1} a_{2} \\
& +\left[\left(m^{2}+m\right) B v+(3 m-3) A+(2 m-1) A^{\prime} v\right] \\
& \times a_{2}^{2} v+\left[(3 m-1) a_{1}^{\prime}-(3 p-q)(p-q)\right] a_{2} A v \\
& \left.+\left[2 q-3 p+(3 m-3) a_{1}\right] A v a_{2}^{\prime}\right\} v_{x}^{m-1} \\
& +(m-1) e^{(p-q) x}\left[-(3 m-4) a_{1} a_{2}+3 a_{2}^{\prime}+a_{2}(3 p-2 q)\right] \\
& \times a_{2} v^{2} A v_{x}^{m-2}-(m-1)(m-2) A a_{2}^{3} v^{3} v_{x}^{m-3}+v_{x}^{2} C_{v} \\
& +2 C_{x v} v_{x}+C_{x x}+a_{2}\left(C-v C_{v}\right)+a_{1} C_{x}=0,
\end{aligned}
$$

where the primes and subscripts denote the derivatives and the partial derivatives with respect to the indicated variables, respectively. To vanish all the coefficients of (24), we will have the following overdetermined system:

$$
\begin{gathered}
B^{\prime \prime}=0, \\
{\left[\frac{p}{m}-a_{1}\right] A^{\prime \prime}+2\left[p-q-(m+1) a_{1}\right] B^{\prime}=0,} \\
m\left(2 A^{\prime}+(m+1) B\right) a_{1}^{2}-\left[(m+1) B+2 A^{\prime}\right] a_{1}^{\prime} \\
-\left[A^{\prime \prime} v+2 A^{\prime}+(2 m+3) B^{\prime} v+m B\right] a_{2}^{\prime} \\
+p\left[\frac{2}{m}(p-q)-2 a_{1}\right] A^{\prime} \\
+(p-q)\left[B\left(p-(2 m+1) a_{1}\right)-2 a_{1} A^{\prime}\right]+B q^{2}=0
\end{gathered}
$$




$$
\begin{aligned}
& {[(3 m-2) p-(2 m-1) q] A a_{1}^{2}-\left(m^{2}-m\right) A a_{1}^{3}} \\
& +\left[(4 m-1) A^{\prime} v+2 m(m+1) B v+3(m-1) A\right] a_{1} a_{2} \\
& +\left[-(p-q)\left(\frac{3 m-1}{m} p-q\right)+(3 m-1) a_{1}^{\prime}\right] A a_{1} \\
& +\left[-\frac{(2 m+1) A^{\prime} v+(m-1) A}{m} p\right. \\
& \left.-2(p-q)\left(A+A^{\prime} v+(2 m+1) B v\right)\right] a_{2} \\
& -\left(2 A^{\prime} v+2 A+(m+1) B v\right) a_{2}^{\prime} \\
& +\left[\frac{p(p-q)^{2}}{m}-a_{1}^{\prime \prime}+(2 q-3 p) a_{1}^{\prime}\right] A=0, \\
& {[(6 m-5) p-(4 m-3) q] A v a_{1} a_{2}-(3 m-2)(m-1)} \\
& \times A a_{1}^{2} a_{2} v+\left[\left(m^{2}+m\right) B v+(3 m-3) A+(2 m-1) A^{\prime} v\right] \\
& \times a_{2}^{2} v+\left[(3 m-1) a_{1}^{\prime}-(3 p-q)(p-q)\right] a_{2} A v \\
& +\left[2 q-3 p+(3 m-3) a_{1}\right] A v a_{2}^{\prime}=0, \\
& {\left[-(3 m-4) a_{1} a_{2}+3 a_{2}^{\prime}+a_{2}(3 p-2 q)\right] a_{2} v^{2} A=0,} \\
& (m-1)(m-2) A a_{2}^{3} v^{3}=0, \\
& C_{v v}=0, \\
& C_{x v}=0 \text {, } \\
& C_{x x}+a_{2}\left(C-v C_{v}\right)+a_{1} C_{x}=0 .
\end{aligned}
$$

For general $m$, it is apparent that $B(u)=a u+b$ and $a_{2}=0$. Substituting $B(u)$ into the second of the above system, we arrive at

$$
\frac{p}{m} A^{\prime \prime}+2(p-q) a-a_{1}\left[A^{\prime \prime}+2(m+1) a\right]=0 .
$$

To solve (26), we can derive three possibilities: $a_{1}(x)=s \neq p / m$, $a_{1}(x)=p / m$, and $a_{1}(x) \neq$ constant.

Assume that the diffusion coefficient $P(u)$ takes power or exponential forms. From (16), without loss of generality, it is reasonable to consider the following four cases: (i) $A(v)=$ $k_{1} v^{2}, B(v)=k_{2} v$; (ii) $A(v)=k_{1} v, B(v)=k_{2}$; (iii) $A(v)=k_{1}$, $B(v)=k_{2}$; and (iv) $A(v)=v^{k}(k \neq 0,1), B(v)=0$, where $k_{1}$ and $k_{2}$ are arbitrary constants.

Case $1\left(a_{1}(x)=s \neq p / m\right)$. In this case, we can derive $A^{\prime \prime}=$ $2 m a[p-q-s(m+1)] /(m s-p)$. Correspondingly, there exist the following cases: (a) $A(v)=(m a[p-q-s(m+1)] /(m s-p)) v^{2}$, $B(v)=a v$; (b) $A(v)=k_{1} v, B(v)=k_{2}$; and (c) $A(v)=k_{1}$,
$B(v)=k_{2}$. If $A(v)=(m a[p-q-s(m+1)] /(m s-p)) v^{2}, B(v)=$ $a v$, the third and fourth equations are simplified as

$$
\begin{aligned}
& \left(m^{2}+m\right) s^{2}-(p-q)(2 m+1) s+(p-q)^{2}=0 \\
& {\left[\left(m^{2}-m\right) s^{2}-(p-q)(2 m-1) s+(p-q)^{2}\right]} \\
& \quad \times(p-q-s(m+1))=0 .
\end{aligned}
$$

Then, we derive $s=(p-q) / m$ or $s=(p-q) /(m+1)$. However, if $s=(p-q) /(m+1)$, function $A(v)$ turns to zero, which should be omitted. By the similar calculation, we obtain $a_{1}(x)=(p-q) / m$ or $a_{1}(x)=(p-q) /(m-1)$ with case (b), while we derive $a_{1}(x)=(p-q) / m$ with case (c). Therefore, we have results listed as the 1-6th entries in Table 1 with Case 1.

Case $2\left(a_{1}(x)=p / m\right)$. In this case, $(26)$ becomes $2 a(p-q-$ $((m+1) p / m))=0$, which implies $a=0$ or $q=-p / m$. When $q \neq-p / m$, we have $a=0$; the corresponding solution is listed as the seventh entry in Table 1 with arbitrary $A(v)$. And results in the case of $q=-p / m$ are presented as the $8-11$ th entries in Table 1.

Case $3\left(a_{1}(x) \neq\right.$ constant). In this case, we can derive

$$
\begin{gathered}
\frac{p}{m} A^{\prime \prime}+2(p-q) a=0, \\
A^{\prime \prime}+2(m+1) a=0,
\end{gathered}
$$

which arrives at $a=0$ or $a \neq 0, q=-p / m$. When $a=0$, it is easy to solve out $A(v)=c v+e$. Without loss of generality, we consider two cases (a) $A(v)=k_{1} v, B(v)=k_{2}$, and (b) $A(v)=$ $k_{1}, B(v)=k_{2}$. If $A(v)=k_{1} v, B(v)=k_{2}$, we can formulate $a_{1}(x)$ according to the third equation of (25). Then, substituting $a_{1}(x)$ into the fourth equation, we have the following condition:

$$
\begin{gathered}
(2 m+1) p^{2}+\left(2 m^{2}-m-1\right) p q-(2 m+1) m q^{2}=0, \\
(m+1) p+(m+1) m q=0 .
\end{gathered}
$$

Therefore, for general $m$, we have $q=-p / m$. So, we derive the corresponding result as the 12-13th entries in the Table 1. By similar calculation, we obtain solution listed as 14-15th entries in Table 1 with the case of $A(v)=k_{1}, B(v)=k_{2}$. When $a \neq 0, q=-p / m$, it is apparent that $A(v)=-(m+1) a v^{2}+c v+e$; we assume $A(v)=-(m+1) a v^{2}, B(v)=a v(m \neq-1)$ without loss of generality. Then, we substitute $A(v)$ and $B(v)$ into the third equation of (25), acquiring $a_{1}(x)=p[c(m+$ 1) $\left.e^{-p x / m}-1\right] / m\left[c m e^{-p x / m}-1\right]$. After translation transformation of $x$, we have $a_{1}(x)=p\left[(m+1) e^{-p x / m}-1\right] / m\left[m e^{-p x / m}-\right.$ $1]$, which can be seen as the same case with the former one. Substituting $a_{1}(x)$ into the fourth equation, we find it identical spontaneously. The corresponding result is listed as the 16th entry in Table 1.

Finally, we have all the solutions listed in the Table 1 with case of $l=2$ for general $m$ including the special relation between $p$ and $q$, where the unknown functions are given as 


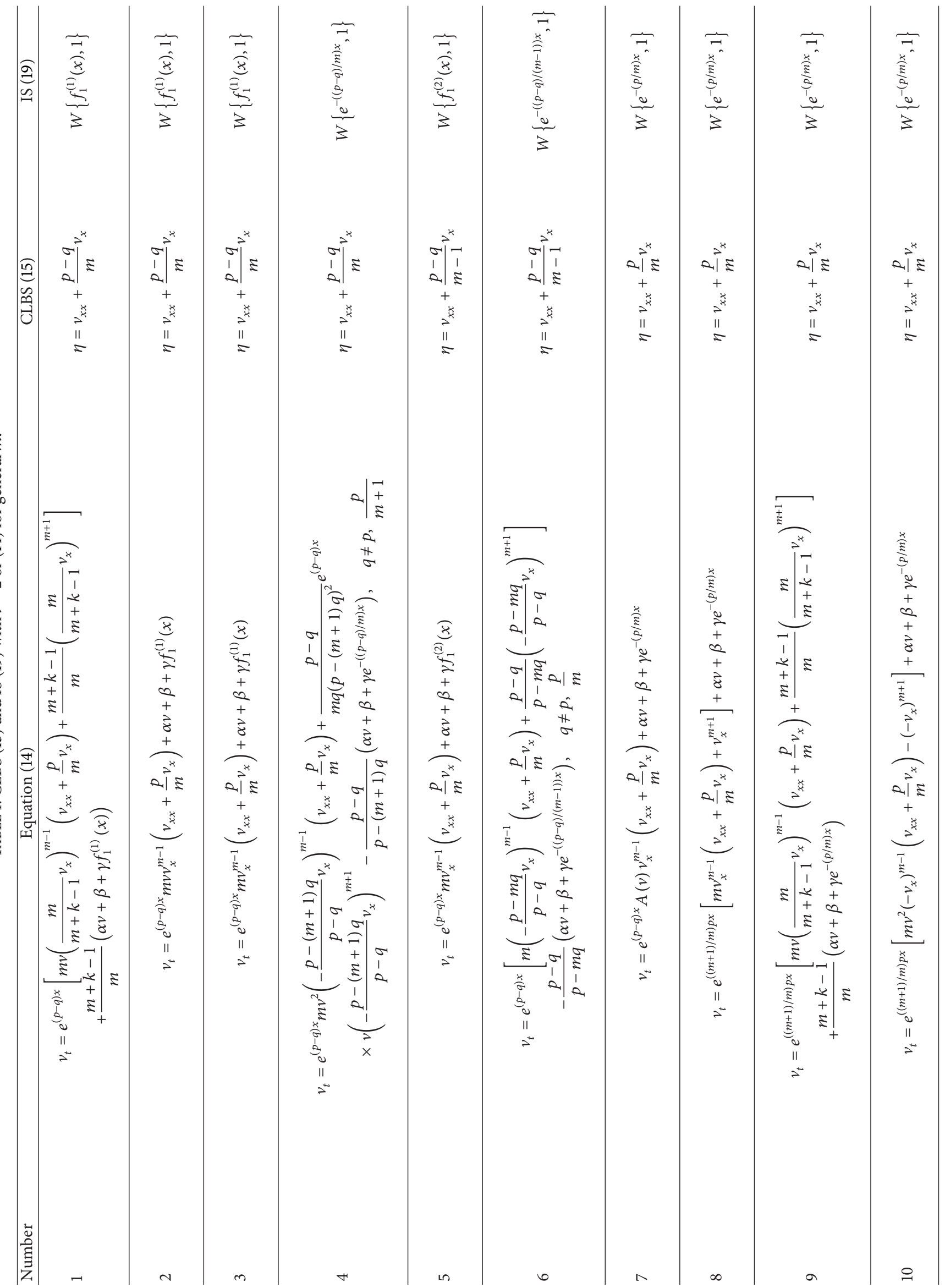




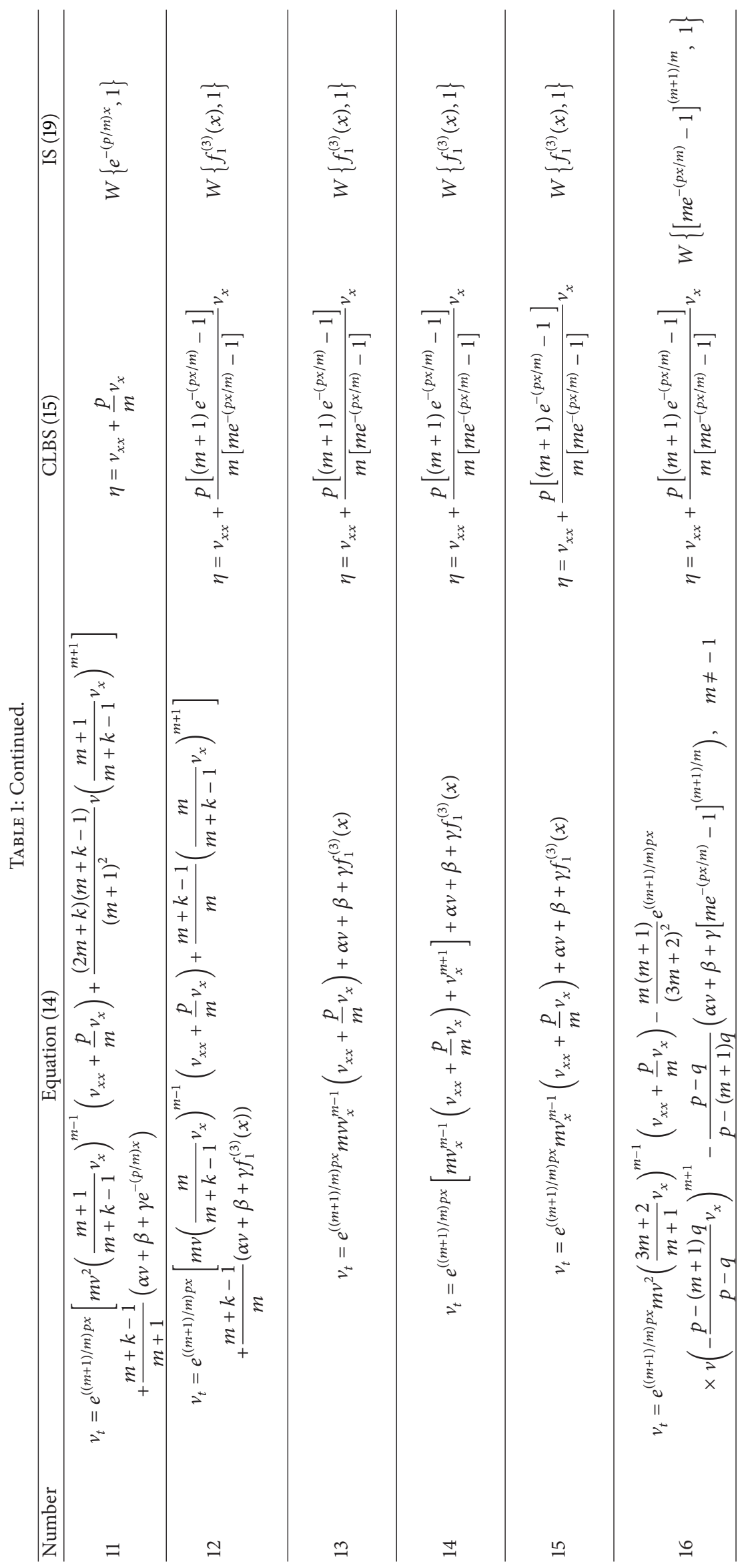


$f_{1}^{(1)}(x)=e^{-((p-q) / m) x}$, for $q \neq p$, and $f_{1}^{(1)}(x)=x$, for $q=p$; $f_{1}^{(2)}(x)=e^{-((p-q) /(m-1)) x}$, for $q \neq p$, and $f_{1}^{(2)}(x)=x$, for $q=p$; $f_{1}^{(3)}(x)=\left[m e^{-p x / m}-1\right]^{(m+1) / m}$, for $m \neq-1$ and $f_{1}^{(3)}(x)=$ $\ln \left(1+e^{p x}\right)$, for $m=-1$.

For special $m$, it is noted that we can combine some terms in (24). This leads to new overdetermined system which is different from (25). Therefore, there exist new forms of (14) admitting CLBS (15) and IS (19) with $l=2$, which are not included in Table 1.

For $m=3$, it follows from (24) that the coefficients in (14) and (15) satisfy

$$
\begin{gathered}
B^{\prime \prime}=0, \\
\left(\frac{p}{3}-a_{1}\right) A^{\prime \prime}+2\left(p-q-4 a_{1}\right) B^{\prime}=0, \\
3\left(2 A^{\prime}+4 B\right) a_{1}^{2}-\left(4 B+2 A^{\prime}\right) a_{1}^{\prime} \\
-\left(A^{\prime \prime} v+2 A^{\prime}+9 B^{\prime} v+3 B\right) a_{2}^{\prime}+p\left[\frac{2}{3}(p-q)-2 a_{1}\right] A^{\prime} \\
+(p-q)\left[B\left(p-7 a_{1}\right)-2 a_{1} A^{\prime}\right]+B q^{2}=0, \\
(7 p-5 q) A a_{1}^{2}-6 A a_{1}^{3}+\left(11 A^{\prime} v+24 B v+6 A\right) a_{1} a_{2} \\
+\left[-(p-q)\left(\frac{8}{3} p-q\right)+8 a_{1}^{\prime}\right] A a_{1} \\
+\left[-\frac{7 A^{\prime} v+2 A}{3} p-2(p-q)\left(A+A^{\prime} v+7 B v\right)\right] a_{2} \\
-\left(2 A^{\prime} v+2 A+4 B v\right) a_{2}^{\prime} \\
+\left[\frac{p(p-q)^{2}}{3}-a_{1}^{\prime \prime}+(2 q-3 p) a_{1}^{\prime}\right] A=0, \\
(13 p-9 q) A v a_{1} a_{2}-14 A a_{1}^{2} a_{2} v+\left(12 B v+6 A+5 A^{\prime} v\right) \\
\times a_{2}^{2} v+\left[8 a_{1}^{\prime}-(3 p-q)(p-q)\right] a_{2} A v \\
+\left(2 q-3 p+6 a_{1}\right) A v a_{2}^{\prime}+C_{v v} e^{-(p-q) x}=0, \\
2 e^{(p-q) x}\left[-5 a_{1} a_{2}+3 a_{2}^{\prime}+a_{2}(3 p-2 q)\right] a_{2} v^{2} A \\
+2 C_{x v}=0, \\
C_{x x}-2 A a_{2}^{3} v^{3}+a_{2}\left(C-v C_{v}\right)+a_{1} C_{x}=0 .
\end{gathered}
$$

Solving the above system by the same approach, we can obtain new results which are presented in Table 2. Similarly, we also list new results in Table 2 for other special $m$.

The unknown functions in Table 2 are given in the following:

$$
\begin{array}{r}
f_{1}^{(4)}(x)=e^{-(p / 2) x}, \quad f_{2}^{(4)}(x)=x e^{-(p / 2) x}, \quad \text { for } q=-\frac{1}{2} p, \\
f_{1}^{(4)}(x)=e^{-((4 p-q) / 9) x}, \quad f_{2}^{(4)}(x)=e^{-((p-q) / 3) x}, \\
\text { for } q \neq-\frac{1}{2} p ;
\end{array}
$$

$$
\begin{aligned}
& f_{1}^{(5)}(x)=e^{-(p / 4) x}, \quad f_{2}^{(5)}(x)=x e^{-(p / 4) x}, \quad \text { for } q=\frac{1}{2} p, \\
& f_{1}^{(5)}(x)=e^{(1 / 2)(-1+\sqrt{-3(p-2 q) /(5 p-6 q))}(p-q) x}, \\
& f_{2}^{(5)}(x)=e^{-(1 / 2)(1+\sqrt{-3(p-2 q) /(5 p-6 q))}(p-q) x}, \\
& \text { for }(p-2 q)(5 p-6 q)<0 \text {, } \\
& f_{1}^{(5)}(x)=e^{-(1 / 2)(p-q) x} \sin \left(\sqrt{\frac{3(p-2 q)}{5 p-6 q}} \frac{p-q}{2} x\right) \text {, } \\
& f_{2}^{(5)}(x)=e^{-(1 / 2)(p-q) x} \cos \left(\sqrt{\frac{3(p-2 q)}{5 p-6 q}} \frac{p-q}{2} x\right), \\
& \text { for }(p-2 q)(5 p-6 q)>0 \text {; } \\
& f_{1}^{(6)}(x)=e^{-(p / 4) x}, \quad f_{2}^{(6)}(x)=x e^{-(p / 4) x}, \quad \text { for } q=\frac{1}{2} p, \\
& f_{1}^{(6)}(x)=e^{-((p-q) / 2) x}, \quad f_{2}^{(6)}(x)=e^{-((p+q) / 6) x}, \quad \text { for } q \neq \frac{1}{2} p ; \\
& f_{1}^{(7)}(x)=e^{-(p / 6) x}, \quad f_{2}^{(7)}(x)=x e^{-(p / 6) x}, \quad \text { for } q=\frac{5}{6} p, \\
& f_{1}^{(7)}(x)=e^{-(p-q) x}, \quad f_{2}^{(7)}(x)=e^{((2 p-3 q) / 3) x}, \\
& \text { for } q \neq \frac{5}{6} p \text {; } \\
& f_{1}^{(8)}(x)=e^{-(p / 2) x}, \quad f_{2}^{(8)}(x)=x e^{-(p / 2) x}, \quad \text { for } q=-\frac{1}{2} p, \\
& f_{1}^{(8)}(x)=e^{-(p / 2) x}, \quad f_{2}^{(8)}(x)=e^{-((p-q) / 3) x}, \quad \text { for } q \neq-\frac{1}{2} p ; \\
& f_{1}^{(9)}(x)=e^{-((p-q) / 2) x}, \quad f_{2}^{(9)}(x)=x e^{-((p-q) / 2) x}, \\
& \text { for } p k-3 q k+4 p-6 q=0 \text {, } \\
& f_{1}^{(9)}(x)=e^{(1 / 2)(-p+q+\sqrt{(-(p-q)(p k-3 q k+4 p-6 q)) /(k+2))} x}, \\
& f_{2}^{(9)}(x)=e^{-(1 / 2)(p-q+\sqrt{(-(p-q)(p k-3 q k+4 p-6 q)) /(k+2))} x}, \\
& \text { for }(p k-3 q k+4 p-6 q)(p-q)(k+2)<0, \\
& =e^{-(1 / 2)(p-q) x} \sin \left(\frac{1}{2} \sqrt{\frac{(p-q)(p k-3 q k+4 p-6 q)}{k+2}} x\right),
\end{aligned}
$$

$f_{2}^{(9)}(x)$

$$
=e^{-(1 / 2)(p-q) x} \cos \left(\frac{1}{2} \sqrt{\frac{(p-q)(p k-3 q k+4 p-6 q)}{k+2} x}\right),
$$

for $(p k-3 q k+4 p-6 q)(p-q)(k+2)>0$;

$$
f_{1}^{(10)}(x)=e^{-((p-q) / 2) x}, \quad f_{2}^{(10)}(x)=x e^{-((p-q) / 2) x},
$$

for $p-q=\frac{k+1}{2 k+5}(p+q)$, 


$$
\begin{aligned}
& f_{1}^{(10)}(x)=e^{-((p-q) / 2) x}, \quad f_{2}^{(10)}(x)=e^{-((k+1)(p+q) /(4 k+10)) x}, \\
& \text { for } p-q \neq \frac{k+1}{2 k+5}(p+q) \text {; } \\
& f_{1}^{(11)}(x)=e^{-(p / 2) x}, \quad f_{2}^{(11)}(x)=x e^{-(p / 2) x}, \quad \text { for } q=\frac{1}{2} p, \\
& f_{1}^{(11)}(x)=e^{-(p / 2) x}, \quad f_{2}^{(11)}(x)=e^{-(p-q) x}, \quad \text { for } q \neq \frac{1}{2} p ; \\
& f_{1}^{(12)}(x)=e^{-(p / 3) x}, \quad f_{2}^{(12)}(x)=x e^{-(p / 3) x}, \quad \text { for } q=\frac{2}{3} p, \\
& f_{1}^{(12)}(x)=e^{-((p+q) / 5) x}, \quad f_{2}^{(12)}(x)=e^{-((3 p-2 q) / 5) x}, \\
& \text { for } q \neq \frac{2}{3} p \\
& f_{1}^{(13)}(x)=e^{-(p-q) x}, \quad f_{2}^{(13)}(x)=x e^{-(p-q) x}, \\
& \text { for } s=2 p-2 q \text {, } \\
& f_{1}^{(13)}(x)=e^{-(p-q) x}, \quad f_{2}^{(13)}(x)=e^{(p-q-s) x}, \\
& \text { for } s \neq 2 p-2 q \text {; } \\
& f_{1}^{(14)}(x)=e^{\sqrt{(s / 2 p)} x}, \quad f_{1}^{(14)}(x)=e^{-\sqrt{(s / 2 p) x}}, \\
& \text { for } s p<0 \text {, } \\
& f_{1}^{(14)}(x)=\sin \left(\sqrt{-\frac{s}{2 p} x}\right), \quad f_{1}^{(14)}(x)=\cos \left(\sqrt{-\frac{s}{2 p} x}\right) \text {, } \\
& \text { for } s p>0 \text {; } \\
& f_{1}^{(15)}(x)=\int \frac{e^{p x}}{s e^{q x}-1} d x
\end{aligned}
$$

When $l=3,4$, by similar calculation, we can derive the classification result listed in Table 3, where $f_{2}^{(16)}(x)=e^{-((p-q) / 2) x}$, for $q \neq p$, and $f_{2}^{(16)}(x)=x$, for $q=p$; $f_{2}^{(17)}(x)=x e^{-(p-q) x}, f_{3}^{(15)}(x)=x^{2} e^{-(p-q) x}$, for $(2 p-3 q)$ $(p-q)=0$, and $f_{2}^{(17)}(x)=e^{(-p+q+\sqrt{-(2 p-3 q)(p-q)}) x}, f_{3}^{17}(x)=$ $e^{(-p+q \sqrt{-(2 p-3 q)(p-q)}) x}$, for $(2 p-3 q)(p-q)<0$, and $f_{2}^{(17)}(x)=$ $e^{-(p-q) x} \sin (\sqrt{(2 p-3 q)(p-q)} x), \quad f_{3}^{(17)}(x)=e^{-(p-q) x}$ $\cos (\sqrt{(2 p-3 q)(p-q)} x)$, for $(2 p-3 q)(p-q)>0 ; f_{1}^{(18)}(x)=$ $e^{-(p / 3) x}, f_{2}^{(18)}(x)=x e^{-(p / 3) x}, f_{3}^{(18)}(x)=x^{2} e^{-(p / 3) x}$, for $q=$ $(2 / 3) p$, and $f_{1}^{(18)}(x)=e^{-(p-q) x}, f_{2}^{(18)}(x)=e^{-((3 p-2 q) / 5) x}$, $f_{3}^{(18)}(x)=e^{-((p+q) / 5) x}$, for $q \neq(2 / 3) p$.

For $l=5$, we find that the overdetermining system is inconsistent. So, there are no CLBSs (15) and ISs (19) for (14).

\section{CLBS (13) and Reductions of (4)}

For (16), we can transfer the CLBS (15) of (14) into CLBS (13) of (4); that is,

$$
u=f(v)=\int \frac{1}{A(v)} \exp \left[\int m \frac{B(v)}{A(v)} d v\right] d v
$$

$$
\begin{aligned}
& P(u)=\frac{A(g(u))}{m}\left[f^{\prime}(g(u))\right]^{1-m}, \\
& Q(x, u)=f^{\prime}(g(u)) C(r, g(u)),
\end{aligned}
$$

where $v=g(u)$ is the inverse function of $u=f(v)$ as referred above. Hence, the GVSS of (4) can be derived from the GVSS (21) with the transformation $u=f(v)$. Here, the GVSS (21) is defined on IS (19) determined by the linear ODE $\eta=0$, where $\eta$ is given by CLBS (15). The coefficient functions $C_{i}(t)(i=1,2, \ldots, l)$ are determined by a finite-dimensional dynamic system. For simplification, we just give some examples to illustrate our approach. Here, we pointed out that the selection of examples is random.

Example 5. Equation

$$
\begin{aligned}
u_{t}= & e^{-p x}\left(e^{p x} u^{2} u_{x}^{-1}\right)_{x}+\alpha \ln (u) u \\
& +\left[\beta+\gamma \ln \left(1+e^{p x}\right)\right] u
\end{aligned}
$$

admits the CLBS

$$
\sigma=[\ln (u)]_{x x}-\frac{p}{e^{p x}+1}[\ln (u)]_{x} .
$$

The corresponding GVSS is given by

$$
u(x, t)=e^{C_{1}(t)+C_{2}(t) \ln \left(1+e^{p x}\right)},
$$

where $C_{1}(t)$ and $C_{2}(t)$ satisfy the 2-dimensional dynamic system

$$
\begin{gathered}
C_{1}^{\prime}=\frac{1}{C_{2}}+1+\alpha C_{1}+\beta, \\
C_{2}^{\prime}=\alpha C_{2}+\gamma .
\end{gathered}
$$

When $\alpha=0$, we have

$$
\begin{gathered}
C_{1}=(\beta+1) t+\frac{\ln \left(\gamma t+c_{2}\right)}{\gamma}+c_{1}, \\
C_{2}=\gamma t+c_{2},
\end{gathered}
$$

with arbitrary constants $c_{1}$ and $c_{2}$. If $\alpha \neq 0$, we have

$$
\begin{gathered}
C_{1}=-\frac{\beta+1}{\alpha}+\frac{1}{\gamma}+\frac{c_{2} \alpha \ln \left(c_{2} \alpha-\gamma e^{-\alpha t}\right)}{\gamma^{2}}+c_{1}, \\
C_{2}=c_{2} e^{\alpha t}-\frac{\gamma}{\alpha},
\end{gathered}
$$

with arbitrary constants $c_{1}$ and $c_{2}$.

Example 6. Equation

$$
\begin{aligned}
u_{t}= & e^{-p x}\left(e^{p x} u^{-2} u_{x}^{2}\right)_{x}-2 s+\alpha u \\
& +\left[\beta \sin \left(\sqrt{-\frac{s}{2 p}} x\right)+\gamma \cos \left(\sqrt{-\frac{s}{2 p}} x\right)\right] u^{3 / 2}
\end{aligned}
$$




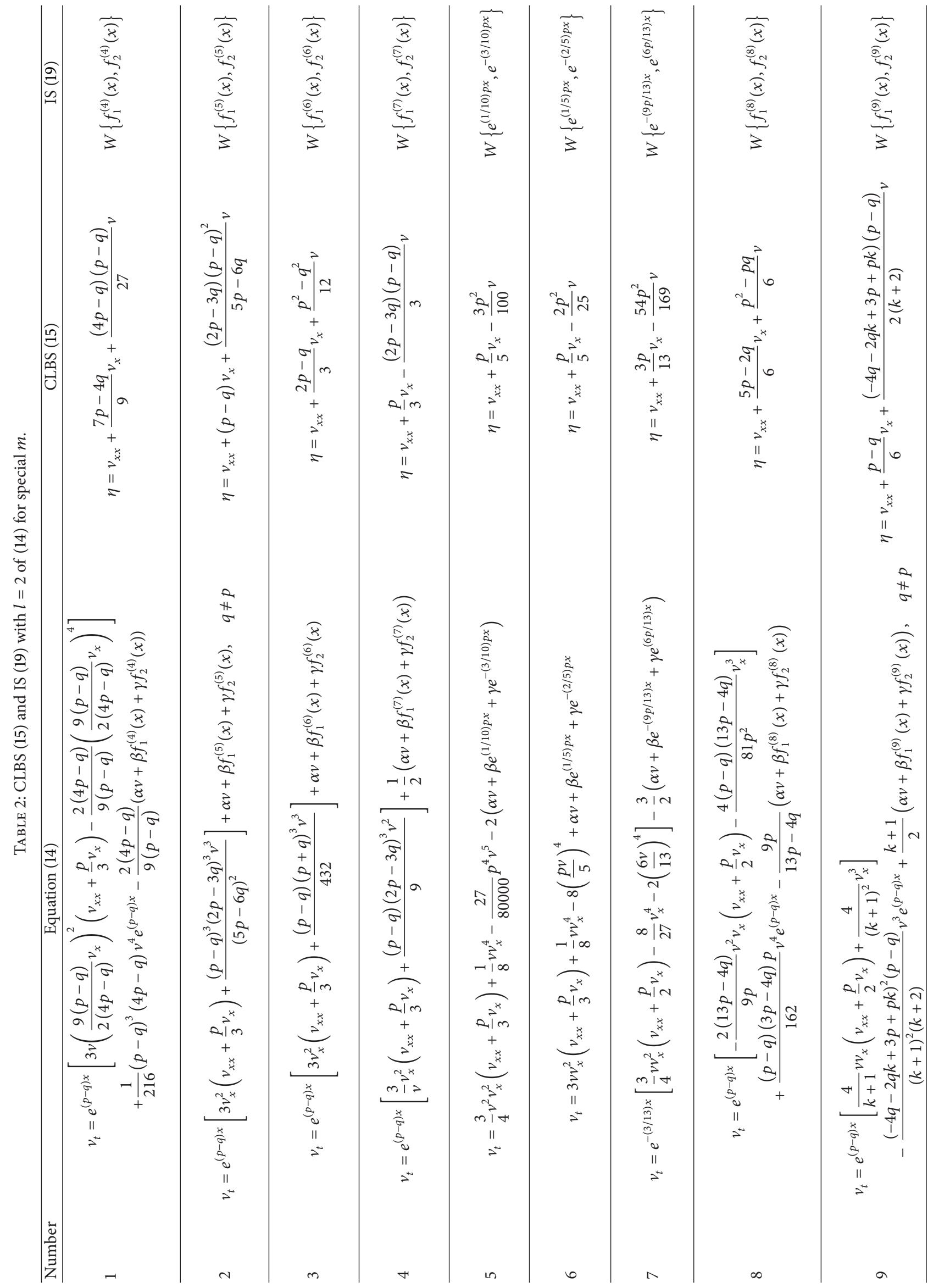




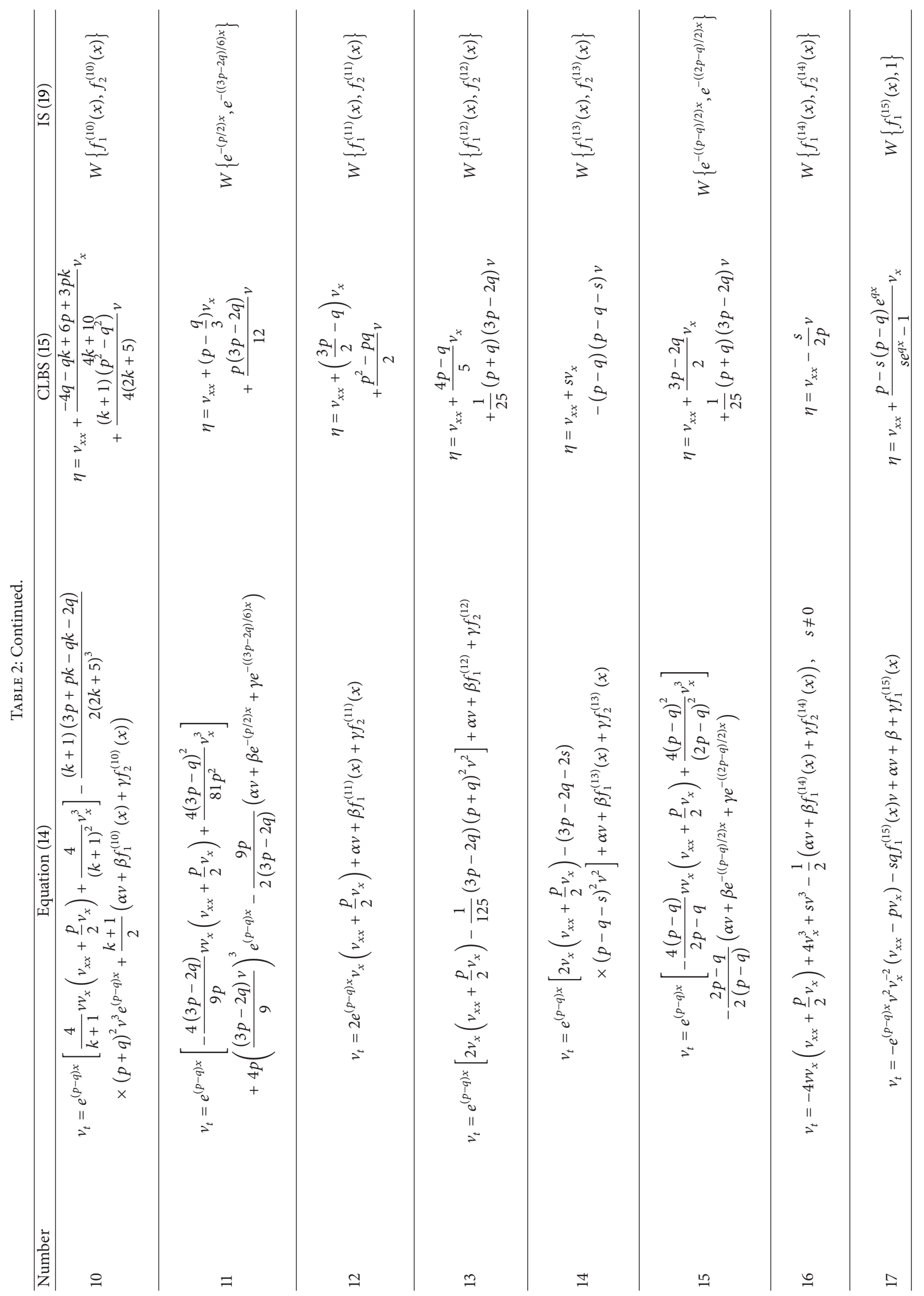




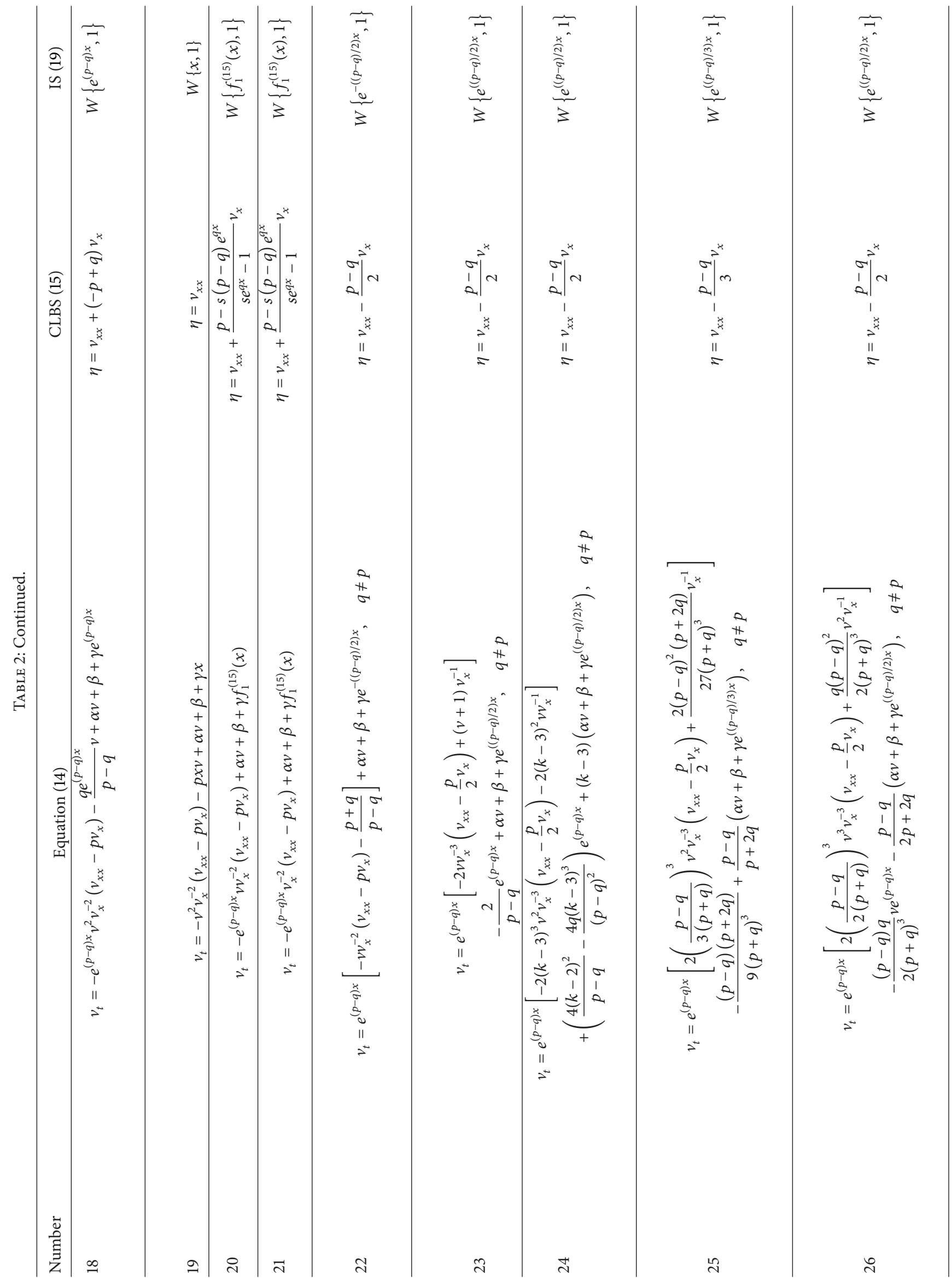




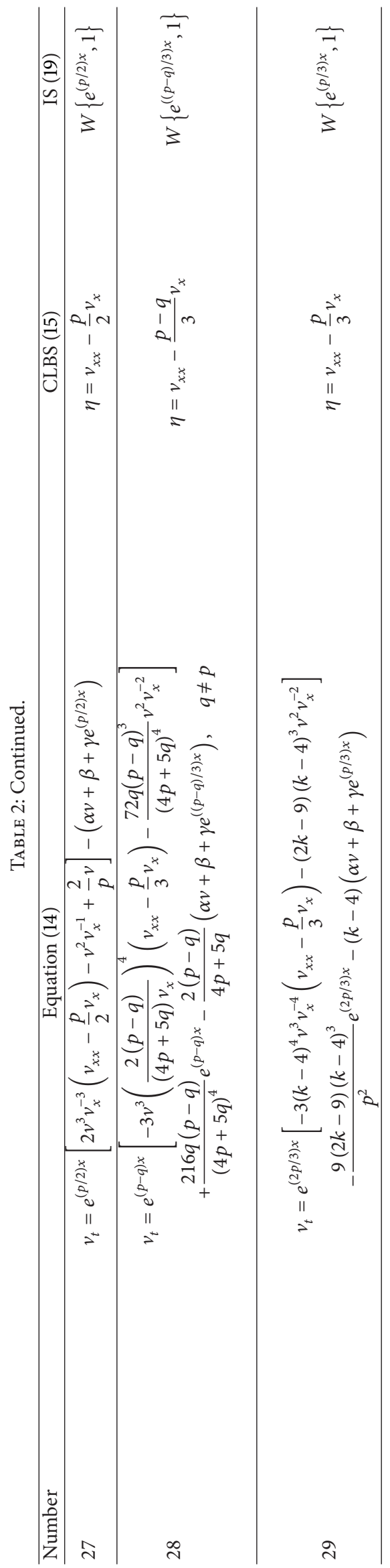




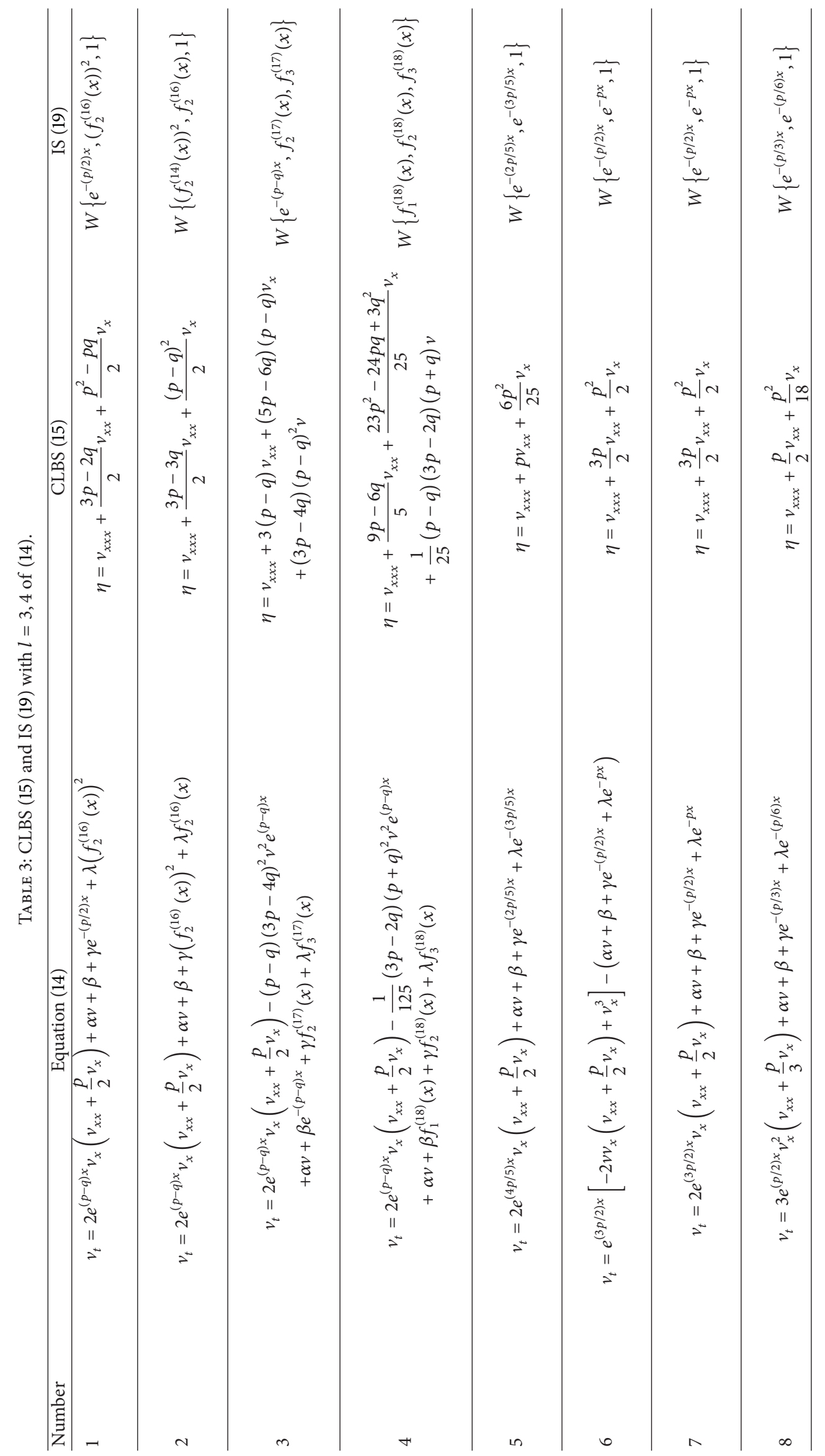




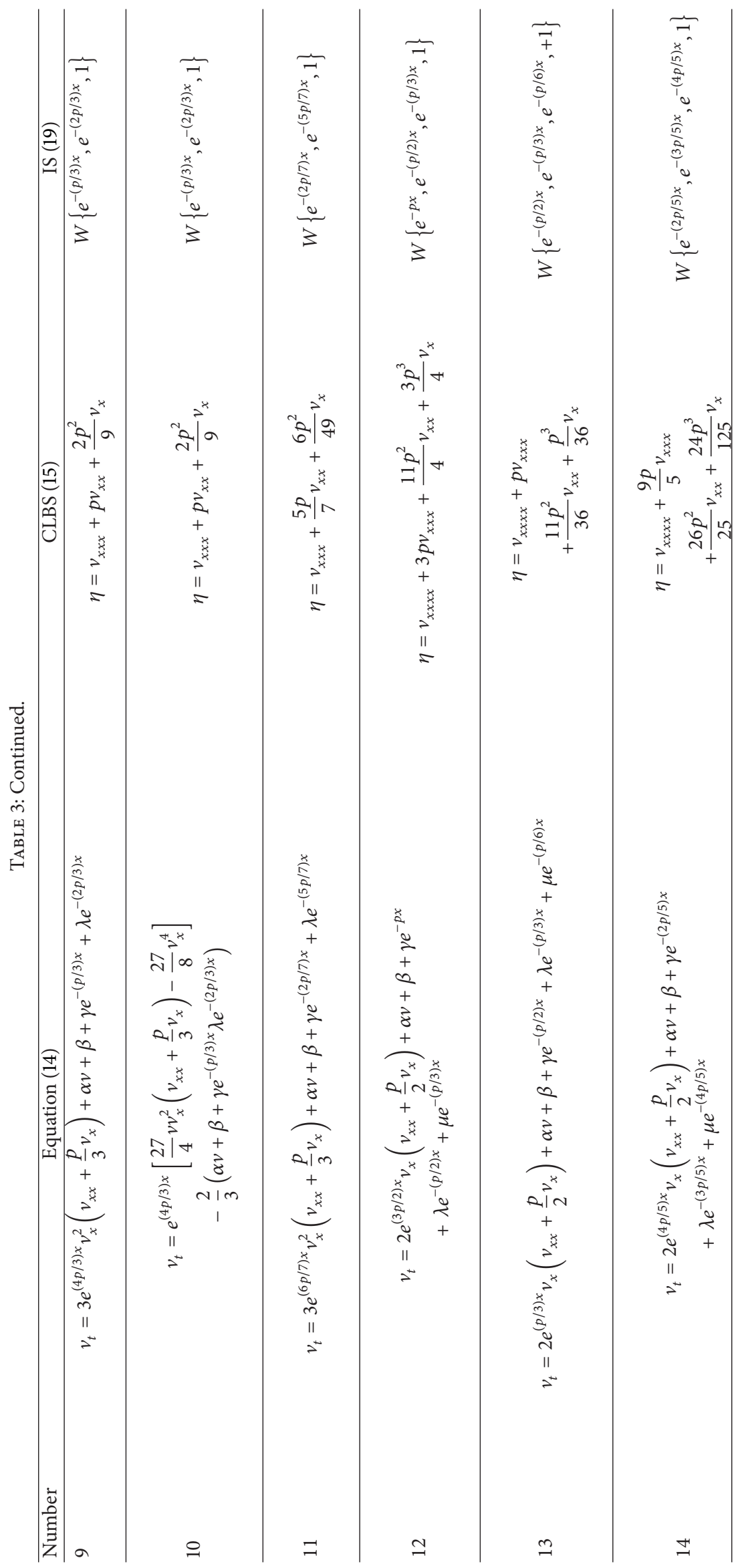


with $s \neq 0$ admits the CLBS

$$
\sigma=\left(u^{-1 / 2}\right)_{x x}-\frac{s}{2 p} u^{-1 / 2} .
$$

The corresponding GVSS is given by

$u(x, t)=\left[C_{1}(t) \sin \left(\sqrt{-\frac{s}{2 p}} x\right)+C_{2}(t) \cos \left(\sqrt{-\frac{s}{2 p}} x\right)\right]^{-2}$,

where $C_{1}(t)$ and $C_{2}(t)$ satisfy the 2-dimensional dynamic system

$$
\begin{aligned}
& C_{1}^{\prime}=\left(C_{1}^{2}+C_{2}^{2}\right)\left[-4 C_{2}\left(-\frac{s}{2 p}\right)^{3 / 2}+s C_{1}\right]-\frac{1}{2}\left(\alpha C_{1}+\beta\right), \\
& C_{2}^{\prime}=\left(C_{1}^{2}+C_{2}^{2}\right)\left[4 C_{1}\left(-\frac{s}{2 p}\right)^{3 / 2}+s C_{2}\right]-\frac{1}{2}\left(\alpha C_{2}+\gamma\right) .
\end{aligned}
$$

Example 7. Equation

$$
\begin{aligned}
u_{t}= & e^{-q x}\left(e^{p x} u^{3} u_{x}^{-2}\right)_{x}+\frac{q}{(p+q)^{2}} u e^{(p-q) x}+\alpha u \\
& +\left(\beta+\gamma e^{((p-q) / 2) x}\right) u^{(3 p+q) / 2(p+q)}
\end{aligned}
$$

with $p \neq q,-q$ admits the CLBS

$$
\sigma=\left(u^{-((p-q) / 2(p+q))}\right)_{x x}-\frac{p-q}{2}\left(u^{-((p-q) / 2(p+q))}\right)_{x} .
$$

The corresponding GVSS is given by

$$
u(x, t)=\left[C_{1}(t)+C_{2}(t) e^{((p-q) / 2) x}\right]^{-(2(p+q) /(p-q))},
$$

where $C_{1}(t)$ and $C_{2}(t)$ satisfy the 2-dimensional dynamic system

$$
\begin{aligned}
& C_{1}^{\prime}=-\frac{q(p-q) C_{1}^{3}}{2(p+q)^{3} C_{2}^{2}}-\frac{p-q}{2(p+q)}\left(\alpha C_{1}+\beta\right), \\
& C_{2}^{\prime}=-\frac{q(p-q) C_{1}^{2}}{2(p+q)^{3} C_{2}}-\frac{p-q}{2(p+q)}\left(\alpha C_{2}+\gamma\right) .
\end{aligned}
$$

Example 8. Equation

$$
u_{t}=e^{-(2 p / 3) x}\left(e^{p x} u_{x}^{2}\right)_{x}-\frac{p^{3}}{27} e^{(p / 3) x} u^{2}
$$

admits the CLBS

$$
\sigma=u_{x x x}+p u_{x x}+\frac{p^{2}}{3} u_{x}+\frac{p^{3}}{27} u
$$

The corresponding GVSS is given by

$$
u(x, t)=\left[C_{1}(t)+C_{2}(t) x+C_{3}(t) x^{2}\right] e^{-(p / 3) x},
$$

where $C_{1}(t), C_{2}(t)$, and $C_{3}(t)$ satisfy the 3 -dimensional dynamic system

$$
\begin{gathered}
C_{1}^{\prime}=-\frac{1}{3}\left(4 p C_{1} C_{3}+p C_{2}^{2}-12 C_{2} C_{3}\right), \\
C_{2}^{\prime}=-\frac{1}{3}\left(8 p C_{2} C_{3}-24 C_{3}^{2}\right), \\
C_{3}^{\prime}=-\frac{8 p C_{3}^{2}}{3} .
\end{gathered}
$$

This system has the following exact solution:

$$
\begin{gathered}
C_{1}=\frac{1}{12} \frac{c_{2}^{2} p^{2}+12 c_{1} p^{2} \sqrt{8 p t+c_{3}}+\left(6561+18 c_{2} p\right) \ln \left[8 p t+c_{3}\right]}{p^{2}\left(8 p t+c_{3}\right)}, \\
C_{2}=\frac{p c_{2}+9 \ln \left[8 p t+c_{3}\right]}{p\left(8 p t+c_{3}\right)}, \\
C_{3}=\frac{3}{8 p t+c_{3}} .
\end{gathered}
$$

\section{Example 9. Equation}

$$
u_{t}=e^{(p / 2) x}\left(e^{p x} u^{-3} u_{x}^{2}\right)_{x}+\alpha u+\left(\beta+\gamma e^{-(p / 2) x}+\lambda e^{-p x}\right) u^{2}
$$

admits the CLBS

$$
\sigma=\left(\frac{1}{u}\right)_{x x x}+\frac{3 p}{2}\left(\frac{1}{u}\right)_{x x}+\frac{p^{2}}{2}\left(\frac{1}{u}\right)_{x}
$$

The corresponding GVSS is given by

$$
u(x, t)=\frac{1}{C_{1}(t)+C_{2}(t) e^{-(p / 2) x}+C_{3}(t) e^{-p x}},
$$

where $C_{1}(t), C_{2}(t)$, and $C_{3}(t)$ satisfy the 3 -dimensional dynamic system

$$
\begin{gathered}
C_{1}^{\prime}=-\frac{p^{3}}{8} C_{2}^{3}+\frac{p^{3}}{2} C_{1} C_{2} C_{3}-\left(\alpha C_{1}+\beta\right), \\
C_{2}^{\prime}=-\frac{p^{3}}{4} C_{2}^{2} C_{3}+p^{3} C_{1} C_{3}^{2}-\left(\alpha C_{2}+\gamma\right), \\
C_{3}^{\prime}=-\left(\alpha C_{3}+\lambda\right) .
\end{gathered}
$$

Example 10. Equation

$$
\begin{aligned}
u_{t}= & e^{(p / 3) x}\left(e^{p x} u^{-4} u_{x}^{3}\right)_{x}+\alpha u \\
& +\left(\beta+\gamma e^{-(p / 3) x}+\lambda e^{-(2 p / 3) x}\right) u^{5 / 3}
\end{aligned}
$$

admits the CLBS

$$
\sigma=\left(u^{-2 / 3}\right)_{x x x}+p\left(u^{-2 / 3}\right)_{x x}+\frac{2 p^{2}}{9}\left(u^{-2 / 3}\right)_{x} .
$$


The corresponding GVSS is given by

$$
u(x, t)=\left[C_{1}(t)+C_{2}(t) e^{-(p / 3) x}+C_{3}(t) e^{-(2 p / 3) x}\right]^{-3 / 2},
$$

where $C_{1}(t), C_{2}(t)$, and $C_{3}(t)$ satisfy the 3 -dimensional dynamic system

$$
\begin{aligned}
& C_{1}^{\prime}=\frac{1}{24} p^{4} C_{2}^{2}\left(4 C_{1} C_{3}-C_{2}^{2}\right)-\frac{2}{3}\left(\alpha C_{1}+\beta\right), \\
& C_{2}^{\prime}=\frac{1}{6} p^{4} C_{2} C_{3}\left(4 C_{1} C_{3}-C_{2}^{2}\right)-\frac{2}{3}\left(\alpha C_{2}+\gamma\right), \\
& C_{3}^{\prime}=\frac{1}{6} p^{4} C_{3}^{2}\left(4 C_{1} C_{3}-C_{2}^{2}\right)-\frac{2}{3}\left(\alpha C_{3}+\lambda\right) .
\end{aligned}
$$

Example 11. Equation

$$
\begin{aligned}
u_{t}= & e^{(p / 2) x}\left(e^{p x} u_{x}^{2}\right)_{x}+\alpha u+\beta+\gamma e^{-p x}+\lambda e^{-(p / 2) x} \\
& +\mu e^{-(3 p / 2) x}
\end{aligned}
$$

admits the CLBS

$$
\sigma=u_{x x x x}+3 p u_{x x x}+\frac{11 p^{2}}{4} u_{x x}+\frac{3 p^{3}}{4} u_{x}
$$

The corresponding GVSS is given by

$$
\begin{aligned}
u(x, t)= & C_{1}(t)+C_{2}(t) e^{-p x}+C_{3}(t) e^{-(p / 2) x} \\
& +C_{4}(t) e^{-(3 p / 2) x},
\end{aligned}
$$

where $C_{1}(t), C_{2}(t), C_{3}(t)$, and $C_{4}(t)$ satisfy the following 4dimensional dynamic system:

$$
\begin{gathered}
C_{1}^{\prime}=-\frac{p^{3}}{2} C_{2} C_{3}+\alpha C_{1}+\beta, \\
C_{2}^{\prime}=-\frac{9 p^{3}}{2} C_{2} C_{4}+\alpha C_{2}+\gamma, \\
C_{3}^{\prime}=-p^{3} C_{2}^{2}-\frac{3 p^{3}}{2} C_{3} C_{4}+\alpha C_{3}+\lambda, \\
C_{4}^{\prime}=-\frac{9 p^{3}}{2} C_{4}^{2}+\alpha C_{4}+\mu .
\end{gathered}
$$

\section{Conclusions and Discussions}

In this paper, we have applied the CLBS method with ISs to study (4). The transformed equations (14) admitting CLBSs (15) are listed in Tables 1, 2, and 3. The corresponding reduced equations of the resulting equations are finite-dimensional dynamic systems defined on $W_{l}$. Some concrete examples are illustrated in Section 4. Generally speaking, these reductions cannot be obtained in the frameworks within the Lie point symmetry method and the nonclassical symmetry method. Of course, we mention that the asymptotical behavior, blowup, extinction, and geometric properties for these finitedimensional dynamic systems are worthy of further study.
For NLDEs, we find that the CLBS method plays a key role in the study of their asymptotical behavior, blow-up, extinction, and geometric properties because of the diversity of solutions obtained by this method. Although this is an effective and complete method, there are still some important problems to be studied. How to study higher-dimensional NLDEs and systems via the CLBS method? How to deal with the initial value problems by means of the CLBS method? Is it possible to apply the CLBS method to other types of evolution equations?

\section{Conflict of Interests}

The authors declare that there is no conflict of interests regarding the publication of this paper.

\section{Acknowledgments}

This work is supported by the National Natural Science Foundation of China (Grant no. 11371323) and the Natural Science Foundation of Zhejiang province (Grant no. Y6100611, Q12A010084).

\section{References}

[1] L. V. Ovsiannikov, Group Analysis of Differential Equations, Academic Press, New York, NY, USA, 1982.

[2] N. H. Ibragimov, Transformation Groups Applied To Mathematical Physics, Reidel, Boston, Mass, USA, 1985.

[3] G. W. Bluman and S. Kumei, Symmetries and Differential Equations, Springer, New York, NY, USA, 1989.

[4] P. J. Olver, Applications of Lie Groups To Differential Equations, Springer, New York, NY, USA, 2nd edition, 1993.

[5] N. H. Ibragimov, CRC Handbook of Lie Group Analysis of Differential Equations, vol. 1, CRC Press, Boca Raton, Fla, USA, 1994.

[6] G. W. Bluman and S. Anco, Symmetry and Integration Methods For Differential Equations, Springer, New York, NY, USA, 2002.

[7] G. W. Bluman, A. Cheviakov, and S. Anco, Applications of Symmetry Methods To Partial Differential Equations, Springer, New York, NY, USA, 2010.

[8] G. W. Bluman and J. D. Cole, "The general similarity solution of the heat equation," Journal of Applied Mathematics and Mechanics, vol. 18, pp. 1025-1042, 1969.

[9] P. J. Olver and P. Rosenau, "Group invariant solutions of differential equations," SIAM Journal on Applied Mathematics, vol. 47, no. 2, pp. 263-278, 1987.

[10] P. J. Olver and P. Rosenau, "The construction of special solutions to partial differential equations," Physics Letters A, vol. 114, no. 3, pp. 107-112, 1986.

[11] M. C. Nucci, "Iterating the nonclassical symmeteries method," Physica D, vol. 78, no. 1-2, pp. 124-134, 1994.

[12] P. A. Clarkson and M. D. Kruskal, "New similarity reductions of the Boussinesq equation," Journal of Mathematical Physics, vol. 30, no. 10, pp. 2201-2213, 1989.

[13] S. Y. Lou and H.-C. Ma, "Non-Lie symmetry groups of $(2+1)-$ dimensional nonlinear systems obtained from a simple direct method," Journal of Physics A, vol. 38, no. 7, pp. L129-L137, 2005.

[14] R. Z. Zhdanov, "Conditional Lie-Backlund symmetry and reduction of evolution equations," Journal of Physics A, vol. 28, no. 13, article no. 027, pp. 3841-3850, 1995. 
[15] A. S. Fokas and Q. M. Liu, "Nonlinear interaction of traveling waves of nonintegrable equations," Physical Review Letters, vol. 72, no. 21, pp. 3293-3296, 1994.

[16] A. S. Fokas and Q. M. Liu, "Generalized conditional symmetries and exact solutions of non-integrable equations," Theoretical and Mathematical Physics, vol. 99, no. 2, pp. 571-582, 1994.

[17] V. A. Galaktionov, "Invariant subspaces and new explicit solutions to evolution equations with quadratic nonlinearities," Proceedings of the Royal Society of Edinburgh A, vol. 125, no. 2, pp. 225-246, 1995.

[18] S. R. Svirshchevskii, "Lie-Bäcklund symmetries of linear ODEs and generalized separation of variables in nonlinear equations," Physics Letters A, vol. 199, no. 5-6, pp. 344-348, 1995.

[19] V. A. Galaktionov and S. Svirshchevski, Exact Solutions and Invariant Subspaces of Nonlinear Partial Differential Equations in Mechanics and Physics, Chapman \& Hall, London, UK, 2007.

[20] J. R. King, "Exact similarity solutions to some nonlinear diffusion equations," Journal of Physics A, vol. 23, no. 16, article 17, pp. 3681-3697, 1990.

[21] C. Sophocleous, "Linearizing mappings for certain nonlinear diffusion equations," Journal of Physics A, vol. 31, no. 29, pp. 6293-6307, 1998.

[22] C. Sophocleous, "Symmetries and form-preserving transformations of generalised inhomogeneous nonlinear diffusion equations," Physica A, vol. 324, no. 3-4, pp. 509-529, 2003.

[23] C. Sophocleous, "Classification of potential symmetries of generalised inhomogeneous nonlinear diffusion equations," Physica A, vol. 320, pp. 169-183, 2003.

[24] C. Sophocleous, "Further transformation properties of generalised inhomogeneous nonlinear diffusion equations with variable coefficients," Physica A, vol. 345, no. 3-4, pp. 457-471, 2005.

[25] R. O. Popovych and N. M. Ivanova, "New results on group classification of nonlinear diffusion-convection equations," Journal of Physics A, vol. 37, no. 30, pp. 7547-7565, 2004.

[26] M. L. Gandarias and M. S. Bruzón, "Solutions through nonclassical potential symmetries for a generalized inhomogeneous nonlinear diffusion equation," Mathematical Methods in the Applied Sciences, vol. 31, no. 7, pp. 753-767, 2008.

[27] C. Qu, "Group classification and generalized conditional symmetry reduction of the nonlinear diffusion-convection equation with a nonlinear source," Studies in Applied Mathematics, vol. 99, no. 2, pp. 107-136, 1997.

[28] Q. Changzheng, "Exact solutions to nonlinear diffusion equations obtained by a generalized conditional symmetry method," IMA Journal of Applied Mathematics, vol. 62, no. 3, pp. 283-302, 1999.

[29] L. N. Ji and C. Z. Qu, "Conditional Lie Bäcklund symmetries and solutions to $(\mathrm{n}+1)$-dimensional nonlinear diffusion equations," Journal of Mathematical Physics, vol. 48, no. 10, Article ID 103509, 2007.

[30] C. Qu, L. Ji, and L. Wang, "Conditional lie bäcklund symmetries and sign-invariants to quasi-linear diffusion equations," Studies in Applied Mathematics, vol. 119, no. 4, pp. 355-391, 2007.

[31] C. Qu and C. Zhu, "Classification of coupled systems with twocomponent nonlinear diffusion equations by the invariant subspace method," Journal of Physics A, vol. 42, no. 47, Article ID 475201, 2009.

[32] L. Ji, C. Qu, and Y. Ye, "Solutions and symmetry reductions of the n-dimensional non-linear convection-diffusion equations," IMA Journal of Applied Mathematics, vol. 75, no. 1, pp. 17-55, 2010.
[33] L. Ji, "Conditional Lie-bäcklund symmetries and solutions of inhomogeneous nonlinear diffusion equations," Physica A, vol. 389, no. 24, pp. 5655-5661, 2010.

[34] L. Ji, "Conditional Lie-Bäcklund symmetries and functionally generalized separable solutions to the generalized porous medium equations with source," Journal of Mathematical Analysis and Applications, vol. 389, no. 2, pp. 979-988, 2012.

[35] E. A. Saied, "Classification of the extended symmetries of the inhomogeneous nonlinear diffusion equation," Journal of the Physical Society of Japan, vol. 64, no. 4, pp. 1092-1097, 1995.

[36] E. A. Saied and M. M. Hussein, "New classes of similarity solutions of the inhomogeneous nonlinear diffusion equations," Journal of Physics A, vol. 27, no. 14, article 15, pp. 4867-4874, 1994.

[37] E. A. Saied, "The nonclassical solution of the inhomogeneous nonlinear diffusion equation," Applied Mathematics and Computation, vol. 98, no. 2-3, pp. 103-108, 1999.

[38] J. Crank, The Mathematics of Diffusion, Oxford University Press, Oxford, UK, 1975.

[39] J. D. Murray, Mathematical Biology, Springer, Berlin, Germany, 1989. 


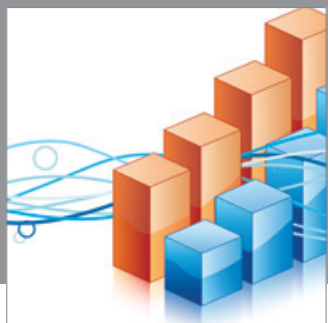

Advances in

Operations Research

mansans

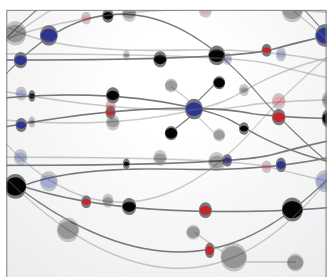

The Scientific World Journal
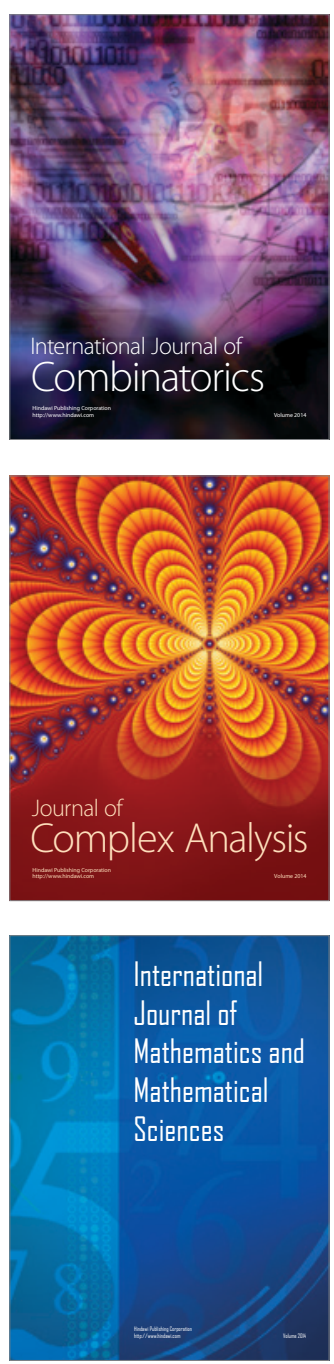
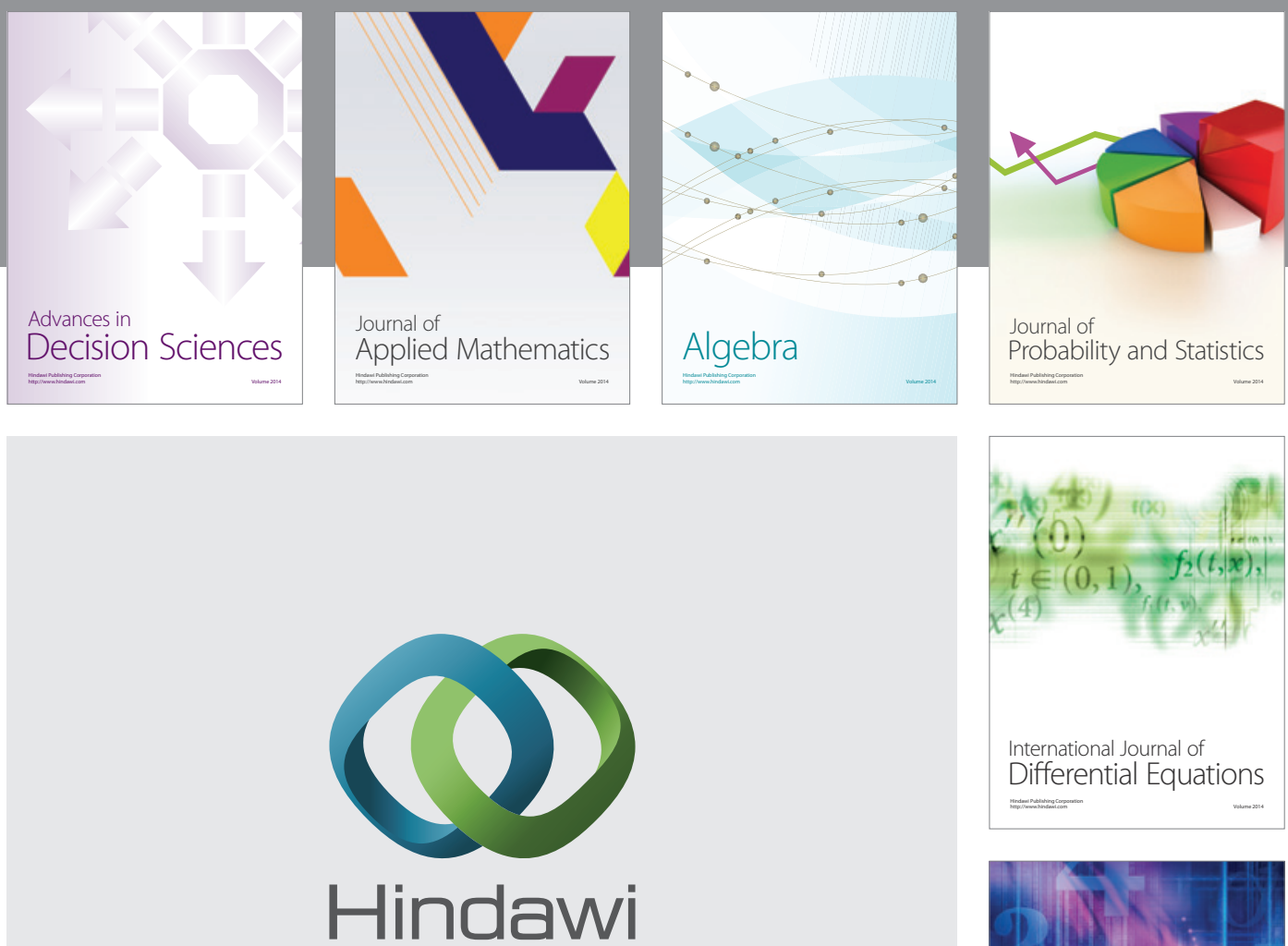

Submit your manuscripts at http://www.hindawi.com
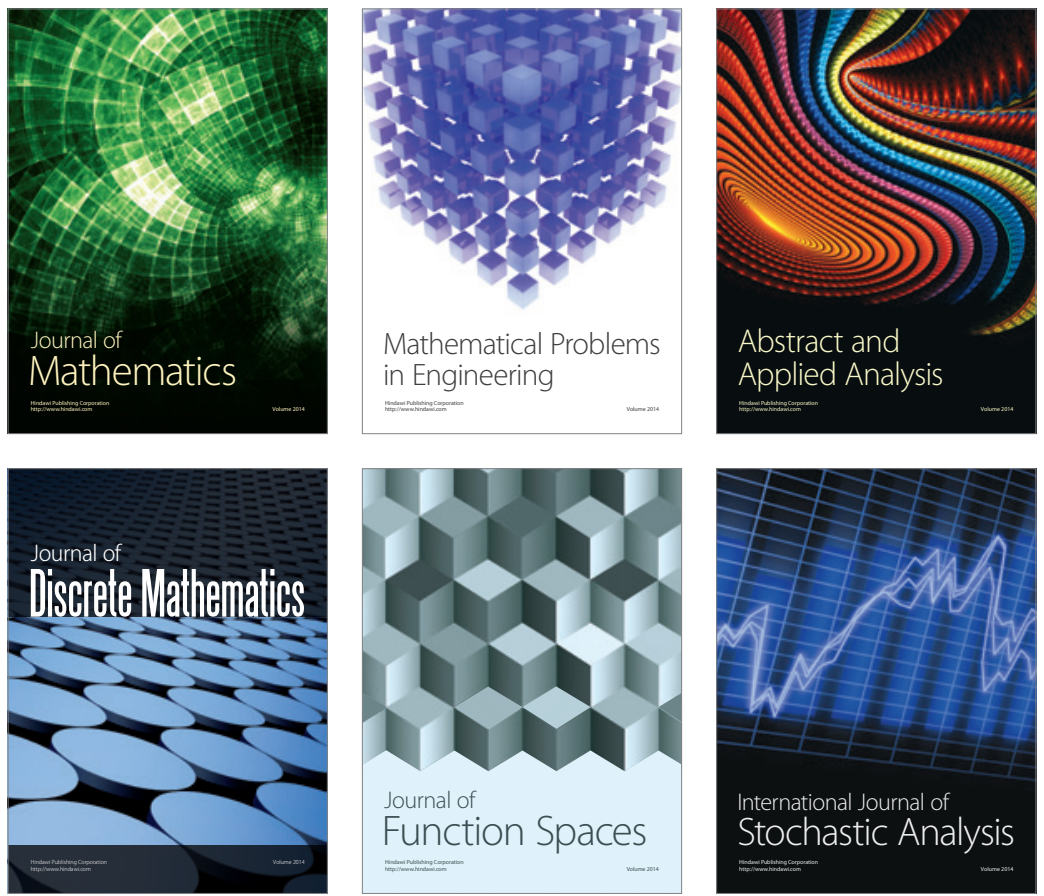

Journal of

Function Spaces

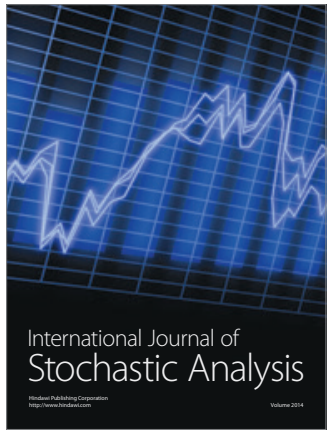

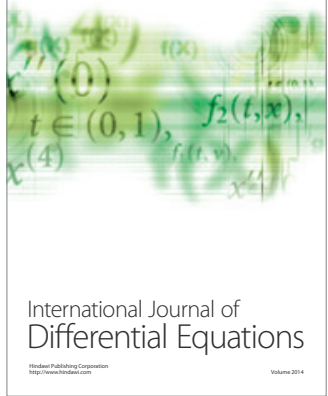
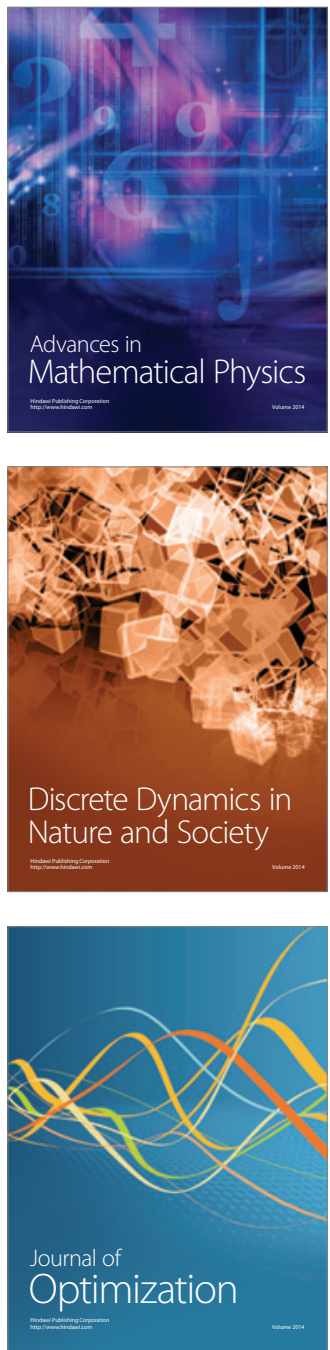